Review

\title{
Symmetry in Generating Functions
}

\section{Shigeru Watanabe}

The University of Aizu, Ikki-machi Tsuruga, Aizu-Wakamatsu City, Fukushima 965-8580, Japan; E-Mail: sigeru-w@u-aizu.ac.jp.

Received: 23 February 2010 / Accepted: 16 March 2010 / Published: 19 March 2010

\begin{abstract}
Generating functions play important roles in theory of orthogonal polynomials. In particular, it is important to consider generating functions that have symmetry. This paper is a survey on generating functions that define unitary operators. First, classical generating functions that define unitary operators are discussed. Next, group theoretical approach to generating functions that have unitarity are discussed.
\end{abstract}

Keywords: Generating function; Orthogonal polynomial; Zonal spherical function

Classification: MSC 43A90 22E30 33C45 33C50 46E20

\section{Introduction}

This paper deals with generating functions that define unitary operators. Problems of this kind were discussed first by Bargmann [1]. He constructed a unitary operator given by an integral operator whose kernel is a generating function of the Hermite polynomials. He also gave a similar construction for the Laguerre polynomials without proof, and noticed as follows ([1], p.203). "It is worth noting that a similar interpretation may be given to other classical generating functions."

We turned our interest to the Gegenbauer polynomials which give the zonal spherical functions on the pair $(S O(n), S O(n-1))$, and in [2] we showed that a similar construction is possible for them. Following this, in [3] we gave a similar construction for the zonal spherical functions on the pair $(U(n), U(n-1))$. On the other hand, A. Essadiq and A. Intissar gave a $q$-analog of Laguerre's case (the result is introduced in [4]), and Essadiq [4] gave a $q$-analog of Gegenbauer's case.

In the two cases that we constructed, we should remark that there is a similarity in the forms of the generating functions. We shall give an explanation in detail. Let $\mathbf{N}_{0}$ be the set of nonnegative integers, 
and let $B$ be the unit open disk $|z|<1$ in $\mathbf{C}$. It is known that the set of all the zonal spherical functions on $(S O(n), S O(n-1))$ is parametrized by $\mathbf{N}_{0}$. Denote the set by $\left\{\varphi_{m} \mid m \in \mathbf{N}_{0}\right\}$. Further, we denote by $d_{m}$ the degree of the representation corresponding to $\varphi_{m}$. Then, the generating function given as the integral kernel of the unitary operator that we constructed is written as a series of the following form:

$$
\sum_{m \in \mathbf{N}_{0}} d_{m} \varphi_{m}(g) z^{m}, \quad g \in S O(n), z \in B
$$

On the other hand, it is known that the set of all the zonal spherical functions on $(U(n), U(n-1))$ is parametrized by $\mathbf{N}_{0}^{2}$. As in the first case, we define $\left\{\varphi_{m} \mid m \in \mathbf{N}_{0}^{2}\right\}$ and $d_{m}\left(m \in \mathbf{N}_{0}^{2}\right)$. Then, the generating function in this case is written as a series of the following form:

$$
\sum_{m \in \mathbf{N}_{0}^{2}} d_{m} \varphi_{m}(g) z^{m}, \quad g \in U(n), z \in B^{2}
$$

where $z^{m}=z_{1}^{m_{1}} z_{2}^{m_{2}}$ for $z=\left(z_{1}, z_{2}\right)$ and $m=\left(m_{1}, m_{2}\right)$.

In this way, the question naturally arises whether similar situations occur in the case of general Gelfand pair $(G, K)$ of the compact type. More precisely, let $G$ be a compact group, and let $K$ be a closed subgroup, such that $(G, K)$ is a Gelfand pair. Further, assume that the set of all the zonal spherical functions on $(G, K)$ is parametrized by $\mathbf{N}_{0}^{\ell}$, where $\ell$ is a positive integer. And as in the case above, define $\left\{\varphi_{m} \mid m \in \mathbf{N}_{0}^{\ell}\right\}$ and $d_{m}\left(m \in \mathbf{N}_{0}^{\ell}\right)$. Then our question can be formulated as follows. Does there exist a generating function of the following form such that it is the kernel of an integral operator which is unitary?

$$
\sum_{m \in \mathbf{N}_{0}^{\ell}} d_{m} \varphi_{m}(g) z^{m}, \quad g \in G, z \in B^{\ell}
$$

where $z^{m}=z_{1}^{m_{1}} \cdots z_{\ell}^{m_{\ell}}$ for $z=\left(z_{1}, \ldots, z_{\ell}\right)$ and $m=\left(m_{1}, \ldots, m_{\ell}\right)$. For this question, we proposed the following theorem in [5].

Theorem 1 Let $G$ be a compact connected Lie group, and let $K$ be a closed subgroup of $G$. If the pair $(G, K)$ is a Riemannian symmetric pair of rank $\ell$ such that $G / K$ is simply connected, then there exists a generating function of the form of (1) which defines a unitary operator.

We should remark that there is a difference between our viewpoint and that of Bargmann. In fact, Bargmann was interested in an operator solution of a commutation rule, which Fock introduced in [6]. Bargmann constructed a function space $\mathcal{F}$ on which Fock's solution is realized, and a unitary operator to study the connection between the space $\mathcal{F}$ and the usual $L^{2}$-space. In his discussion, he used the well known generating function of the Hermite polynomials, but he did not use their other properties. That is, he derived from the unitarity of the operator that the Hermite polynomials form a complete orthogonal system. In contrast, we positively used not only the well known generating functions of the orthogonal polynomials, but also their orthogonality and completeness, and showed the unitarity of the operators.

The purpose of this paper is to present a survey on generating functions that define unitary operators. In Section 3, we give an outline of Bargmann's discussion on the Hermite polynomials. In Section 4, we deal with the case of the Laguerre polynomials. Bargmann gave the construction for them, but he did not 
give its proof. In this section, we shall give our proof that borrows Bargmann's viewpoint. In Sections 5 and 6 , we shall give surveys on the case of the Gegenbauer polynomials, and the case of the zonal spherical functions on the pair $(U(n), U(n-1))$, respectively. In Section 7, we shall give an outline of the proof of Theorem 1 .

\section{Notation and Preliminaries}

We will explain the notation and the terms which will be used throughout this paper.

\subsection{General Notation}

Firstly, we shall use the notation $\mathbf{N}_{0}, \mathbf{R}, \mathbf{C}$ for the set of nonnegative integers, the field of real numbers and the field of complex numbers, respectively. For a fixed positive integer $\ell$, we denote by $\mathbf{C}^{\ell}$ the complex $\ell$-dimensional space, and denote by $\mathbf{N}_{0}^{\ell}$ the set of all multi-indices $m=\left(m_{1}, \ldots, m_{\ell}\right)$ with each $m_{j} \in \mathbf{N}_{0}$. For $m=\left(m_{1}, \ldots, m_{\ell}\right) \in \mathbf{N}_{0}^{\ell}$ and $z=\left(z_{1}, \ldots, z_{\ell}\right) \in \mathbf{C}^{\ell}$ we write $z^{m}=z_{1}^{m_{1}} \cdots z_{\ell}^{m_{\ell}}$. For $z=\left(z_{1}, \ldots, z_{\ell}\right) \in \mathbf{C}^{\ell}$ and $w=\left(w_{1}, \ldots, w_{\ell}\right) \in \mathbf{C}^{\ell}$ we denote $\left(z_{1} w_{1}, \ldots, z_{\ell} w_{\ell}\right) \in \mathbf{C}^{\ell}$ by $z w$. We denote by $B$ the unit open disk in $\mathbf{C}$, and denote by $B^{\ell}$ the open polydisk of $z=\left(z_{1}, \ldots, z_{\ell}\right) \in \mathbf{C}^{\ell}$ with each $z_{j} \in B$. For a subset $A \subset \mathbf{R}$ or $\mathbf{C}$ we define $A^{\ell}$ in the same way. We shall use the notation $[a, b],[a, b)$ and $(a, b)$ for the interval $\{x \in \mathbf{R} \mid a \leq x \leq b\}$, the interval $\{x \in \mathbf{R} \mid a \leq x<b\}$ and the interval $\{x \in \mathbf{R} \mid a<x<b\}$, respectively. For $\zeta \in \mathbf{C}$ let $\operatorname{Re} \zeta$ be the real part of $\zeta$, and $\zeta \mapsto \bar{\zeta}$ the usual conjugation in $\mathbf{C}$. We denote the Gamma function by $\Gamma(x)$, the hypergeometric function by $F(\alpha, \beta ; \gamma ; x)$, the binomial coefficient by $\left(\begin{array}{l}a \\ k\end{array}\right)$ and $\Gamma(a+k) / \Gamma(a)$ by $(a)_{k}$. The minimum value of $a$ and $b$ is denoted by $\min (a, b)$. A function is assumed to be complex-valued.

\subsection{Gelfand Pairs}

A compact group is a topological group whose underlying topology is compact Hausdorff. Let $G$ be a compact group, and let $K$ be a closed subgroup of $G$. A representation of the compact group $G$ means a continuous homomorphism of $G$ to the group $G L(V)$ of invertible linear transformations on a finite-dimensional complex vector space $V$. Since $G$ is compact, for an arbitrary representation of $G$ there exists a $G$-invariant inner product on the representation space $V$, that is, $V$ admits an inner product such that the representation is unitary.

Given an irreducible representation $\rho$ of $G$, let $V_{\rho}$ denote the representation space of $\rho$, and $V_{\rho}^{K}$ the subspace of $V_{\rho}$ consisting of elements $w \in V_{\rho}$ which satisfy $\rho(k) w=w$ for any $k \in K$. If an irreducible representation $\rho$ of $G$ satisfies $V_{\rho}^{K} \neq\{0\}, \rho$ is called a spherical representation of $G$ with respect to the subgroup $K$. Let $\mathcal{D}(G, K)$ denote the set of all the equivalence classes of spherical representations of $G$ with respect to $K$.

Let $d g$ denote the normalized Haar measure on $G$, that is, it has the property that $\int_{G} d g=1$. For two continuous functions $\varphi, \psi$ on $G$ the convolution $\varphi * \psi$ is defined by

$$
(\varphi * \psi)\left(g^{\prime}\right)=\int_{G} \varphi\left(g^{\prime} g^{-1}\right) \psi(g) d g, \quad g^{\prime} \in G
$$


The pair $(G, K)$ is called a Gelfand pair if the convolution algebra of continuous functions on $G$ which are bi-invariant under $K$ is commutative. The pair $(G, K)$ is a Gelfand pair if and only if $\operatorname{dim} V_{\rho}^{K}=1$ for all $\rho \in \mathcal{D}(G, K)$.

Let $(G, K)$ be a Gelfand pair, and let $\rho \in \mathcal{D}(G, K)$. Choose a $G$-invariant inner product $\langle$,$\rangle on the$ representation space $V_{\rho}$, and take an orthonormal basis $\left\{e_{1}\right\}$ of $V_{\rho}^{K}$ with respect to $\langle$,$\rangle . Define$

$$
\varphi(g)=\left\langle e_{1}, \rho(g) e_{1}\right\rangle, \quad g \in G
$$

The function $\varphi$ is called the zonal spherical function associated with $\rho$.

\subsection{Riemannian Symmetric Pairs}

Let $G$ be a compact connected Lie group, and let $K$ be a closed subgroup of $G$. The pair $(G, K)$ is called a Riemannian symmetric pair if there exists an involutive $C^{\infty}$ automorphism $\theta$ of $G$ such that $G_{\theta}^{0} \subset K \subset G_{\theta}$, where $G_{\theta}$ is the set of fixed points of $\theta$ and $G_{\theta}^{0}$ is the identity component of $G_{\theta}$.

Let $(G, K)$ be a Riemannian symmetric pair. Let $\mathfrak{g}$ and $\mathfrak{k}$ be the Lie algebras of $G$ and $K$, respectively. The automorphism of $\mathfrak{g}$ which is the differential of the automorphism $\theta$ of $G$ will also be denoted by $\theta$. Then we have

$$
\mathfrak{k}=\{X \in \mathfrak{g} \mid \theta(X)=X\}
$$

We define the subspace $\mathfrak{m}$ of $\mathfrak{g}$ by

$$
\mathfrak{m}=\{X \in \mathfrak{g} \mid \theta(X)=-X\}
$$

Then the Lie algebra $\mathfrak{g}$ is decomposed into a direct sum of vector spaces as

$$
\mathfrak{g}=\mathfrak{k}+\mathfrak{m}
$$

The subspace $\mathfrak{m}$ is called the canonical complement of the pair $(G, K)$. A maximal abelian subalgebra, which is contained in $\mathfrak{m}$ is called a Cartan subalgebra of the pair $(G, K)$. The Cartan subalgebras have the same dimension, which is called the rank of the Riemannian symmetric pair $(G, K)$.

\section{Generating Function that Defines Unitary Operator-The Case of Hermite Polynomials}

Bargmann [1] constructed a unitary operator given by an integral operator whose kernel is a generating function of the Hermite polynomials. In fact, he was interested in the operator solution

$$
\xi_{k}=\frac{\partial}{\partial \eta_{k}}
$$

of the commutation rule $\left[\xi_{k}, \eta_{k}\right]=1$, which Fock introduced in [6]. He constructed a function space $\mathcal{F}$ on which Fock's solution is realized. This space $\mathcal{F}$ is a Hilbert space associated with the unitary operator introduced above. In this section, we shall summarize Bargmann's discussion, that is: how to construct the space $\mathcal{F}$ and the unitary operator. For the sake of simplicity we only consider the one variable case. 


\section{1. $L^{2}$-Space Associated with the Hermite Polynomials}

The Hermite polynomials $H_{m}(x), m=0,1,2, \ldots$, have the following generating function:

$$
e^{2 x z-z^{2}}=\sum_{m \in \mathbf{N}_{0}} H_{m}(x) \frac{z^{m}}{m !}, \quad z \in \mathbf{C}, x \in \mathbf{R}
$$

Let $d x$ be the Lebesgue measure on $\mathbf{R}$, and let $L^{2}(\mathbf{R}, d x)$ be the Hilbert space of Lebesgue measurable functions $\varphi$ on $\mathbf{R}$ with

$$
\|\varphi\|=\sqrt{\int_{\mathrm{R}}|\varphi(x)|^{2} d x}<\infty
$$

The inner product is given by

$$
(\varphi, \psi)=\int_{\mathrm{R}} \varphi(x) \overline{\psi(x)} d x, \quad \varphi, \psi \in L^{2}(\mathbf{R}, d x)
$$

Set

$$
\phi_{m}(x)=\frac{e^{-x^{2} / 2} H_{m}(x)}{\left(2^{m} m ! \sqrt{\pi}\right)^{1 / 2}}, \quad m \in \mathbf{N}_{0}, x \in \mathbf{R}
$$

Then it is known that $\left\{\phi_{m} \mid m \in \mathbf{N}_{0}\right\}$ is a complete orthonormal system of $L^{2}(\mathbf{R}, d x)$. But, as described in the Introduction, Bargmann did not use this fact in his discussion.

\subsection{Hilbert Space $\mathcal{F}$ of Analytic Functions}

Let $\mathcal{F}$ be the space of entire functions $f$ on $\mathbf{C}$ with

$$
\|f\|=\sqrt{\int_{\mathrm{C}}|f(z)|^{2} \rho\left(|z|^{2}\right) d z}<\infty
$$

where $\rho(t)=\pi^{-1} e^{-t}$, and $d z$ is the Lebesgue measure on $\mathbf{C}$ induced from the identification $\mathbf{C} \cong \mathbf{R}^{2}$. For $f \in \mathcal{F}$, the following inequality holds:

$$
|f(z)| \leq e^{|z|^{2} / 2}\|f\|, \quad z \in \mathbf{C}
$$

which implies that $\mathcal{F}$ is a Hilbert space with inner product

$$
\langle f, g\rangle=\int_{\mathrm{C}} f(z) \overline{g(z)} \rho\left(|z|^{2}\right) d z, \quad f, g \in \mathcal{F}
$$

Notice that Bargmann determined the weight $\rho\left(|z|^{2}\right)$ in order to satisfy the relation

$$
\langle z f, g\rangle=\left\langle f, \frac{d g}{d z}\right\rangle
$$

for functions $f, g$ that do not grow too fast at infinity. Set

$$
u_{m}(z)=\frac{z^{m}}{\sqrt{m !}}, \quad m \in \mathbf{N}_{0}, z \in \mathbf{C}
$$

Then the system $\left\{u_{m} \mid m \in \mathbf{N}_{0}\right\}$ is a complete orthonormal system of $\mathcal{F}$. It also follows from (2) that the space $\mathcal{F}$ has the reproducing kernel. The kernel is given by

$$
g_{w}(z)=\sum_{m \in \mathbf{N}_{0}} u_{m}(\bar{w}) u_{m}(z), \quad z, w \in \mathbf{C}
$$

that is, $g_{w}(z)=e^{\bar{w} z}$ and $f(w)=\left\langle f, g_{w}\right\rangle$ for $f \in \mathcal{F}$ and $w \in \mathbf{C}$. 


\subsection{Unitary Operator Associated with the Hermite Polynomials}

A unitary operator on $L^{2}(\mathbf{R}, d x)$ to $\mathcal{F}$ is defined as follows. First, set

$$
\Phi(z, x)=\pi^{-1 / 4} \exp \left\{-\frac{1}{2}\left(z^{2}+x^{2}\right)+2^{1 / 2} x z\right\}, \quad z \in \mathbf{C}, x \in \mathbf{R}
$$

This has the following expansion:

$$
\Phi(z, x)=\sum_{m \in \mathbf{N}_{0}} u_{m}(z) \phi_{m}(x), \quad z \in \mathbf{C}, x \in \mathbf{R}
$$

which means that the function $\Phi(z, x)$ can be regarded as a generating function of the Hermite polynomials.

For $\varphi \in L^{2}(\mathbf{R}, d x)$ define

$$
(\Phi \varphi)(z)=\int_{\mathrm{R}} \Phi(z, x) \varphi(x) d x, \quad z \in \mathbf{C}
$$

Then $\Phi \varphi \in \mathcal{F}$ and the following holds.

Theorem 2 The operator $\Phi$ on $L^{2}(\mathbf{R}, d x)$ to $\mathcal{F}$ is unitary.

Notice that Bargmann determined the integral kernel $\Phi(z, x)$ in order to satisfy the following conditions: if $\varphi$ is sufficiently smooth and vanishes sufficiently fast at infinity, then

$$
\Phi(\eta \varphi)=z(\Phi \varphi), \quad \Phi(\xi \varphi)=\frac{d(\Phi \varphi)}{d z}
$$

where

$$
\eta=2^{-1 / 2}\left(x-\frac{d}{d x}\right), \quad \xi=2^{-1 / 2}\left(x+\frac{d}{d x}\right)
$$

In what follows, we shall state an outline of Bargmann's proof that $\Phi$ is unitary. Let $\varphi$ be a continuous function on $\mathbf{R}$ with compact support, and let $f=\Phi \varphi$. Define

$$
f_{r}(z)=f(r z), \quad 0<r<1
$$

Then the square of the norm $\left\|f_{r}\right\|$ can be rewritten as follows.

$$
\begin{aligned}
& \left\|f_{r}\right\|^{2}=\left(1+\epsilon^{2}\right) \int_{\mathrm{R}} e^{-\epsilon^{2} s^{2}} N_{\epsilon}(s) d s \\
& N_{\epsilon}(s)=\frac{1}{\sqrt{\pi}} \int e^{-t^{2}} \overline{\varphi(s-\epsilon t)} \varphi(s+\epsilon t) d t
\end{aligned}
$$

where

$$
\epsilon=\left(\frac{1-r^{2}}{1+r^{2}}\right)^{1 / 2}
$$

These relations imply that

$$
\lim _{r \rightarrow 1}\left\|f_{r}\right\|^{2}=\int_{\mathrm{R}}|\varphi(x)|^{2} d x=\|\varphi\|^{2}
$$

It follows from this result that the norms $\left\|f_{r}\right\|, 0<r<1$ are uniformly bounded, which means that $f \in \mathcal{F}$ and

$$
\lim _{r \rightarrow 1}\left\|f_{r}\right\|^{2}=\|f\|^{2}
$$


Remark 1 As stated in the Introduction, Bargmann did not use in his proof the fact that the system $\left\{\phi_{m} \mid m \in \mathbf{N}_{0}\right\}$ is a complete orthonormal system of $L^{2}(\mathbf{R}, d x)$. He conversely defined the function $\phi_{m}$ by $\phi_{m}=\Phi^{-1} u_{m}$, and derived from the unitarity of $\Phi$ that $\left\{\phi_{m} \mid m \in \mathbf{N}_{0}\right\}$ is a complete orthonormal system of $L^{2}(\mathbf{R}, d x)$.

\section{Generating Function that Defines Unitary Operator-The Case of Laguerre Polynomials}

As stated in the Introduction, Bargmann [1] gave a construction similar to Hermite's case for the Laguerre polynomials, but he did not prove this fact. In this section, we shall give our proof for it. Our proof follows Bargmann's viewpoint. That is, we use the well known generating function of the Laguerre polynomials, but we do not use their other properties except some properties that are easily derived from the generating function.

Let $\gamma$ be a positive real number. The Laguerre polynomials $L_{m}^{\gamma}(x), m=0,1,2, \ldots$, have the following generating function:

$$
\frac{\exp \{-x z /(1-z)\}}{(1-z)^{\gamma+1}}=\sum_{m \in \mathbf{N}_{0}} L_{m}^{\gamma}(x) z^{m}, \quad z \in B, 0<x<\infty
$$

The orthogonality relation easily follows from (3) (cf. [7]):

$$
\int_{0}^{\infty} L_{m}^{\gamma}(x) L_{m^{\prime}}^{\gamma}(x) e^{-x} x^{\gamma} d x=\left\{\begin{array}{cc}
\Gamma(m+\gamma+1) / m !, & m=m^{\prime} \\
0, & m \neq m^{\prime}
\end{array}\right.
$$

\section{1. $L^{2}$-Space Associated with the Laguerre Polynomials}

Set

$$
d \nu_{\gamma}(x)=\frac{x^{\gamma}}{\Gamma(\gamma+1)} d x
$$

and let $L^{2}\left((0, \infty), d \nu_{\gamma}(x)\right)$ be the Hilbert space of Lebesgue measurable functions $\varphi$ on the open interval $(0, \infty)$ with

$$
\|\varphi\|=\sqrt{\int_{0}^{\infty}|\varphi(x)|^{2} d \nu_{\gamma}(x)}<\infty
$$

The inner product is given by

$$
(\varphi, \psi)=\int_{0}^{\infty} \varphi(x) \overline{\psi(x)} d \nu_{\gamma}(x), \quad \varphi, \psi \in L^{2}\left((0, \infty), d \nu_{\gamma}(x)\right)
$$

¿From the orthogonality relation of the Laguerre polynomials, we see that the functions $e^{-x / 2} L_{m}^{\gamma}(x), m=$ $0,1,2, \ldots$, form an orthogonal system of $L^{2}\left((0, \infty), d \nu_{\gamma}(x)\right)$. Denote by $\phi_{m}^{\gamma}(x)$ the normalization of $e^{-x / 2} L_{m}^{\gamma}(x)$ with respect to the norm of this space. That is,

$$
\phi_{m}^{\gamma}(x)=\left(\begin{array}{c}
\gamma+m \\
m
\end{array}\right)^{-1 / 2} e^{-x / 2} L_{m}^{\gamma}(x)
$$

Then the system $\left\{\phi_{m}^{\gamma} \mid m \in \mathbf{N}_{0}\right\}$ is an orthonormal system of $L^{2}\left((0, \infty), d \nu_{\gamma}(x)\right)$. 


\subsection{Hilbert Space of Analytic Functions}

Let $d z$ be the Lebesgue measure on $B$ induced from the identification $\mathbf{C} \cong \mathbf{R}^{2}$, and set

$$
d \mu_{\gamma}(z)=\frac{\gamma}{\pi}\left(1-|z|^{2}\right)^{\gamma-1} d z
$$

Let $\mathcal{F}_{\gamma}$ be the space of analytic functions $f$ on $B$ with

$$
\|f\|=\sqrt{\int_{B}|f(z)|^{2} d \mu_{\gamma}(z)}<\infty
$$

In the following, we shall show some properties of the space $\mathcal{F}_{\gamma}$.

Lemma 1 For $f \in \mathcal{F}_{\gamma}$, let $f(z)=\sum_{m \in \mathbf{N}_{0}} \alpha_{m} z^{m}$ be the power series expansion of $f$ in $B$. Then we have

$$
\|f\|^{2}=\sum_{m \in \mathbf{N}_{0}}\left(\begin{array}{c}
\gamma+m \\
m
\end{array}\right)^{-1}\left|\alpha_{m}\right|^{2}
$$

Proof. Let $0<\sigma<1$, and set $B_{\sigma}=\{z \in \mathbf{C}|| z \mid \leq \sigma\}$. It is easy to see that

$$
\int_{B_{\sigma}} z^{m} \overline{z^{m^{\prime}}} d \mu_{\gamma}(z)=\left\{\begin{array}{cc}
\gamma \int_{0}^{\sigma^{2}} t^{m}(1-t)^{\gamma-1} d t, & m=m^{\prime} \\
0, & m \neq m^{\prime}
\end{array}\right.
$$

Hence, we have

$$
\begin{aligned}
\int_{B_{\sigma}}|f(z)|^{2} d \mu_{\gamma}(z) & =\sum_{m \in \mathbf{N}_{0}}\left|\alpha_{m}\right|^{2} \gamma \int_{0}^{\sigma^{2}} t^{m}(1-t)^{\gamma-1} d t \\
& =\sum_{m \in \mathbf{N}_{0}}\left(\begin{array}{c}
\gamma+m \\
m
\end{array}\right)^{-1} c_{m}(\sigma)\left|\alpha_{m}\right|^{2} \leq\|f\|^{2}
\end{aligned}
$$

where

$$
c_{m}(\sigma)=\left(\begin{array}{c}
\gamma+m \\
m
\end{array}\right) \gamma \int_{0}^{\sigma^{2}} t^{m}(1-t)^{\gamma-1} d t
$$

Since $0 \leq c_{m}(\sigma) \leq 1$ and $\lim _{\sigma \rightarrow 1} c_{m}(\sigma)=1$, we obtain

$$
\|f\|^{2}=\lim _{\sigma \rightarrow 1} \int_{B_{\sigma}}|f(z)|^{2} d \mu_{\gamma}(z)=\sum_{m \in \mathbf{N}_{0}}\left(\begin{array}{c}
\gamma+m \\
m
\end{array}\right)^{-1}\left|\alpha_{m}\right|^{2}
$$

(Note that we used 1a. Preliminary remarks in Bargmann [1].)

Lemma 2 Let $f \in \mathcal{F}_{\gamma}$. Then we have

$$
|f(z)| \leq\|f\|\left(1-|z|^{2}\right)^{-(\gamma+1) / 2}, \quad z \in B
$$


Proof. Let $f(z)=\sum_{m \in \mathbf{N}_{0}} \alpha_{m} z^{m}$ be the power series expansion of $f$ in $B$. By the Schwarz inequality and Lemma 1, we have

$$
\begin{aligned}
|f(z)| & =\left|\sum_{m \in \mathbf{N}_{0}}\left\{\left(\begin{array}{c}
\gamma+m \\
m
\end{array}\right)^{-1 / 2} \alpha_{m}\right\}\left\{\left(\begin{array}{c}
\gamma+m \\
m
\end{array}\right)^{1 / 2} z^{m}\right\}\right| \\
& \leq\|f\| \sqrt{\sum_{m \in \mathbf{N}_{0}}\left(\begin{array}{c}
\gamma+m \\
m
\end{array}\right)|z|^{2 m}}=\|f\|\left(1-|z|^{2}\right)^{-(\gamma+1) / 2}
\end{aligned}
$$

which implies our assertion.

It follows from Lemma 2 that $\mathcal{F}_{\gamma}$ is a Hilbert space with inner product

$$
\langle f, g\rangle=\int_{B} f(z) \overline{g(z)} d \mu_{\gamma}(z), \quad f, g \in \mathcal{F}_{\gamma}
$$

Set

$$
u_{m}^{\gamma}(z)=\left(\begin{array}{c}
\gamma+m \\
m
\end{array}\right)^{1 / 2} z^{m}, \quad m \in \mathbf{N}_{0}, z \in B
$$

Then we have

Lemma 3 The system $\left\{u_{m}^{\gamma} \mid m \in \mathbf{N}_{0}\right\}$ is a complete orthonormal system of $\mathcal{F}_{\gamma}$.

Proof. In the same way as in the proof of Lemma 1, we see that $\left\{u_{m}^{\gamma} \mid m \in \mathbf{N}_{0}\right\}$ is an orthonormal system of $\mathcal{F}_{\gamma}$. Next we show the completeness of the system. Let $f$ be an element of $\mathcal{F}_{\gamma}$ with power series expansion $f(z)=\sum_{m \in \mathbf{N}_{0}} \alpha_{m} z^{m}$. From Lemma 1, the power series expansion of $f$ converges with respect to the norm of $\mathcal{F}_{\gamma}$. Hence, we have

$$
\left\langle f, u_{m}^{\gamma}\right\rangle=\left(\begin{array}{c}
\gamma+m \\
m
\end{array}\right)^{-1 / 2} \alpha_{m}
$$

By Lemma 1, we obtain

$$
\|f\|^{2}=\sum_{m \in \mathbf{N}_{0}}\left|\left\langle f, u_{m}^{\gamma}\right\rangle\right|^{2}
$$

which implies the completeness of the system.

It follows from Lemma 2 that $\mathcal{F}_{\gamma}$ has the reproducing kernel. The kernel is given by

$$
g_{w}^{\gamma}(z)=\sum_{m \in \mathbf{N}_{0}} u_{m}^{\gamma}(\bar{w}) u_{m}^{\gamma}(z), \quad z, w \in B
$$

that is, $g_{w}^{\gamma}(z)=(1-\bar{w} z)^{-(\gamma+1)}$ and $f(w)=\left\langle f, g_{w}^{\gamma}\right\rangle$ for $f \in \mathcal{F}_{\gamma}$ and $w \in B$. 


\subsection{Unitary Operator Associated with the Laguerre Polynomials}

A unitary operator on $L^{2}\left((0, \infty), d \nu_{\gamma}(x)\right)$ to $\mathcal{F}_{\gamma}$ is defined as follows. First, set

$$
\Phi(z, x)=\frac{1}{(1-z)^{\gamma+1}} \exp \left\{-\frac{x(1+z)}{2(1-z)}\right\}, \quad z \in B, 0<x<\infty
$$

This has the following expansion:

$$
\Phi(z, x)=\sum_{m \in \mathbf{N}_{0}} u_{m}^{\gamma}(z) \phi_{m}^{\gamma}(x), \quad z \in B, 0<x<\infty
$$

which means that the function $\Phi(z, x)$ is a generating function of the Laguerre polynomials.

For $\varphi \in L^{2}\left((0, \infty), d \nu_{\gamma}(x)\right)$ we define

$$
(\Phi \varphi)(z)=\int_{0}^{\infty} \Phi(z, x) \varphi(x) d \nu_{\gamma}(x), \quad z \in B
$$

Then $\Phi \varphi \in \mathcal{F}_{\gamma}$ and the following holds.

Theorem 3 The operator $\Phi$ on $L^{2}\left((0, \infty), d \nu_{\gamma}(x)\right)$ to $\mathcal{F}_{\gamma}$ is unitary.

In what follows, we shall prove this theorem.

Lemma 4 The operator $\Phi$ is a bounded operator on $L^{2}\left((0, \infty), d \nu_{\gamma}(x)\right)$ to $\mathcal{F}_{\gamma}$.

Proof. Let $\varphi \in L^{2}\left((0, \infty), d \nu_{\gamma}(x)\right)$. It follows from (4), (5) and $\sum_{m \in \mathbf{N}_{0}}\left|u_{m}^{\gamma}(z)\right|^{2}<\infty$ that

$$
(\Phi \varphi)(z)=\left(\varphi, \sum_{m \in \mathbf{N}_{0}} \overline{u_{m}^{\gamma}(z)} \phi_{m}^{\gamma}\right)=\sum_{m \in \mathbf{N}_{0}} u_{m}^{\gamma}(z)\left(\varphi, \phi_{m}^{\gamma}\right), \quad z \in B
$$

Since $\left\{\phi_{m}^{\gamma} \mid m \in \mathbf{N}_{0}\right\}$ is an orthonormal system of $L^{2}\left((0, \infty), d \nu_{\gamma}(x)\right)$, we have $\sum_{m \in \mathbf{N}_{0}}\left|\left(\varphi, \phi_{m}^{\gamma}\right)\right|^{2}$ $<\infty$. Hence, we see that $\Phi \varphi \in \mathcal{F}_{\gamma}$ and

$$
\|\Phi \varphi\|^{2}=\sum_{m \in \mathbf{N}_{0}}\left|\left(\varphi, \phi_{m}^{\gamma}\right)\right|^{2} \leq\|\varphi\|^{2}
$$

which means that $\Phi$ is an operator on $L^{2}\left((0, \infty), d \nu_{\gamma}(x)\right)$ to $\mathcal{F}_{\gamma}$, and bounded.

Lemma 5 The operator $\Phi$ is surjective.

Proof. If we take $\varphi=\phi_{m}^{\gamma}$ in the proof of Lemma 4, we have

$$
\Phi \phi_{m}^{\gamma}=u_{m}^{\gamma}, \quad m \in \mathbf{N}_{0}
$$

Further, $\left\{u_{m}^{\gamma} \mid m \in \mathbf{N}_{0}\right\}$ is a complete orthonormal system of $\mathcal{F}_{\gamma}$, and the operator $\Phi$ is bounded. These facts imply that $\Phi$ is surjective.

Lemma 6 The operator $\Phi$ is injective. 
Proof. Let $\varphi \in L^{2}\left((0, \infty), d \nu_{\gamma}(x)\right)$, and assume that $\Phi \varphi=0$. It is clear that

$$
\int_{0}^{\infty} x^{\gamma} \varphi(x) \exp \left\{-\frac{x(1+z)}{2(1-z)}\right\} d x=0
$$

for each $z \in B$. Let us consider the following linear fractional transformation

$$
w=\frac{1+z}{1-z}
$$

This transformation maps the unit open disk $B$ in the $z$-plane onto $\{w \in \mathbf{C} \mid \operatorname{Re} w>0\}$ in the $w$-plane. Therefore, the condition (7) is equivalent to the following.

$$
\int_{0}^{\infty} e^{-x(\xi+i \eta) / 2} x^{\gamma} \varphi(x) d x=0
$$

for each positive real number $\xi$ and each $\eta \in \mathbf{R}$. Take $\xi=1$ and set

$$
\psi(x)=\left\{\begin{array}{cc}
e^{-x / 2} x^{\gamma} \varphi(x), & x>0 \\
0, & x \leq 0
\end{array}\right.
$$

It follows from (8) that $\psi \in L^{1}(\mathbf{R}, d x)$ and

$$
\int_{-\infty}^{\infty} \psi(x) e^{-i x \eta / 2} d x=0
$$

for all $\eta \in \mathbf{R}$, where $L^{1}(\mathbf{R}, d x)$ is the space of Lebesgue measurable functions $\phi$ on $\mathbf{R}$ with $\int_{-\infty}^{\infty}|\phi(x)| d x$ $<\infty$. Since the Fourier transformation on $L^{1}(\mathbf{R}, d x)$ is injective, by (9) we obtain $\psi=0$. By the definition of $\psi$, we see that $\varphi=0$, which implies that $\Phi$ is injective.

Lemma 7 The operator $\Phi$ is an isometry.

Proof. Let $\varphi \in L^{2}\left((0, \infty), d \nu_{\gamma}(x)\right)$, and set

$$
\Phi \varphi=\sum_{m \in \mathbf{N}_{0}} \alpha_{m} u_{m}^{\gamma}
$$

Then we have

$$
\|\Phi \varphi\|^{2}=\sum_{m \in \mathbf{N}_{0}}\left|\alpha_{m}\right|^{2}
$$

On the other hand, by Lemma 4 and (6), we see that

$$
\Phi \varphi=\sum_{m \in \mathbf{N}_{0}} \alpha_{m} \Phi \phi_{m}^{\gamma}=\Phi\left(\sum_{m \in \mathbf{N}_{0}} \alpha_{m} \phi_{m}^{\gamma}\right)
$$

It follows from this fact and Lemma 6 that

$$
\varphi=\sum_{m \in \mathbf{N}_{0}} \alpha_{m} \phi_{m}^{\gamma}
$$

which implies

$$
\|\varphi\|^{2}=\sum_{m \in \mathbf{N}_{0}}\left|\alpha_{m}\right|^{2}
$$

¿From (10) and (11), we have the assertion.

As a result of these lemmas, we can conclude that $\Phi$ is unitary.

Corollary 1 The system $\left\{\phi_{m}^{\gamma} \mid m \in \mathbf{N}_{0}\right\}$ is complete. 


\section{Generating Function that Defines Unitary Operator - The Case of Gegenbauer Polynomials}

Let $\lambda$ be a positive real number. The Gegenbauer polynomials $C_{m}^{\lambda}(x), m=0,1,2, \ldots$, have the following generating function:

$$
\frac{1}{\left(1-2 x z+z^{2}\right)^{\lambda}}=\sum_{m \in \mathbf{N}_{0}} C_{m}^{\lambda}(x) z^{m}, \quad z \in B,-1<x<1
$$

In this section, we deal with a generating function of $\left\{C_{m}^{\lambda} \mid m \in \mathbf{N}_{0}\right\}$ that defines a unitary operator. For more details, refer to [2].

\section{1. $L^{2}$-Space Associated with the Gegenbauer Polynomials}

Let $L^{2}\left((-1,1),\left(1-x^{2}\right)^{\lambda-1 / 2} d x\right)$ be the Hilbert space of Lebesgue measurable functions $\varphi$ on the open interval $(-1,1)$ with

$$
\|\varphi\|=\sqrt{\int_{-1}^{1}|\varphi(x)|^{2}\left(1-x^{2}\right)^{\lambda-1 / 2} d x}<\infty
$$

The inner product is given by

$$
(\varphi, \psi)=\int_{-1}^{1} \varphi(x) \overline{\psi(x)}\left(1-x^{2}\right)^{\lambda-1 / 2} d x, \quad \varphi, \psi \in L^{2}\left((-1,1),\left(1-x^{2}\right)^{\lambda-1 / 2} d x\right)
$$

As is well known, the Gegenbauer polynomials $C_{m}^{\lambda}(x), m=0,1,2, \ldots$, form a complete orthogonal system of $L^{2}\left((-1,1),\left(1-x^{2}\right)^{\lambda-1 / 2} d x\right)$. Denote by $\phi_{m}^{\lambda}$ the normalization of $C_{m}^{\lambda}$ with respect to the norm of this space. That is,

$$
\phi_{m}^{\lambda}(x)=\sqrt{\frac{2^{2 \lambda-1}(m+\lambda) m !(\Gamma(\lambda))^{2}}{\pi \Gamma(m+2 \lambda)}} C_{m}^{\lambda}(x)
$$

Then the system $\left\{\phi_{m}^{\lambda} \mid m \in \mathbf{N}_{0}\right\}$ is a complete orthonormal system of $L^{2}\left((-1,1),\left(1-x^{2}\right)^{\lambda-1 / 2} d x\right)$.

\subsection{Hilbert Space of Analytic Functions}

For $0<t<1$ we define

$$
\rho_{\lambda}(t)= \begin{cases}\frac{1}{\Gamma(2 \lambda-1)} t^{\lambda-1} \int_{t}^{1} s^{-\lambda}(1-s)^{2 \lambda-2} d s, & \lambda>1 / 2 \\ t^{\lambda-1}\left(\frac{\Gamma(1-\lambda)}{\Gamma(\lambda)}-\frac{1}{\Gamma(2 \lambda-1)} \int_{0}^{t} s^{-\lambda}(1-s)^{2 \lambda-2} d s\right), & 0<\lambda \leq 1 / 2\end{cases}
$$

Let $\mathcal{F}_{\lambda}$ be the space of analytic functions $f$ on $B$ with

$$
\|f\|=\sqrt{\int_{B}|f(z)|^{2} \rho_{\lambda}\left(|z|^{2}\right) d z}<\infty
$$


where $d z$ is the Lebesgue measure on $B$ induced from the identification $\mathbf{C} \cong \mathbf{R}^{2}$. For $f \in \mathcal{F}_{\lambda}$, we have the following inequality

$$
|f(z)| \leq\|f\| \sqrt{\sum_{m \in \mathbf{N}_{0}} \frac{(m+\lambda) \Gamma(m+2 \lambda)}{\pi m !}|z|^{2 m}}, \quad z \in B
$$

which implies that $\mathcal{F}_{\lambda}$ is a Hilbert space with inner product

$$
\langle f, g\rangle=\int_{B} f(z) \overline{g(z)} \rho_{\lambda}\left(|z|^{2}\right) d z, \quad f, g \in \mathcal{F}_{\lambda}
$$

Set

$$
u_{m}^{\lambda}(z)=\sqrt{\frac{(m+\lambda) \Gamma(m+2 \lambda)}{\pi m !}} z^{m}, \quad m \in \mathbf{N}_{0}, z \in B
$$

Then the system $\left\{u_{m}^{\lambda} \mid m \in \mathbf{N}_{0}\right\}$ is a complete orthonormal system of $\mathcal{F}_{\lambda}$. It also follows from (12) that the space $\mathcal{F}_{\lambda}$ has the reproducing kernel. The kernel is given by

$$
g_{w}^{\lambda}(z)=\sum_{m \in \mathbf{N}_{0}} u_{m}^{\lambda}(\bar{w}) u_{m}^{\lambda}(z), \quad z, w \in B
$$

that is, $f(w)=\left\langle f, g_{w}^{\lambda}\right\rangle$ for $f \in \mathcal{F}_{\lambda}$ and $w \in B$.

\subsection{Unitary Operator Associated with the Gegenbauer Polynomials}

A unitary operator on $L^{2}\left((-1,1),\left(1-x^{2}\right)^{\lambda-1 / 2} d x\right)$ to $\mathcal{F}_{\lambda}$ is defined as follows. First, set

$$
\Phi(z, x)=\frac{2^{\lambda-1 / 2} \Gamma(\lambda+1)}{\pi} \frac{1-z^{2}}{\left(1-2 x z+z^{2}\right)^{\lambda+1}}, \quad z \in B,-1<x<1
$$

This has the following expansion:

$$
\Phi(z, x)=\sum_{m \in \mathbf{N}_{0}} u_{m}^{\lambda}(z) \phi_{m}^{\lambda}(x), \quad z \in B,-1<x<1
$$

which means that the function $\Phi(z, x)$ is a generating function of the Gegenbauer polynomials.

For $\varphi \in L^{2}\left((-1,1),\left(1-x^{2}\right)^{\lambda-1 / 2} d x\right)$ we define

$$
(\Phi \varphi)(z)=\int_{-1}^{1} \Phi(z, x) \varphi(x)\left(1-x^{2}\right)^{\lambda-1 / 2} d x, \quad z \in B
$$

Then $\Phi \varphi \in \mathcal{F}_{\lambda}$ and we have

Theorem 4 The operator $\Phi$ on $L^{2}\left((-1,1),\left(1-x^{2}\right)^{\lambda-1 / 2} d x\right)$ to $\mathcal{F}_{\lambda}$ is unitary.

We positively use in our proof the fact that the system $\left\{\phi_{m}^{\lambda} \mid m \in \mathbf{N}_{0}\right\}$ is a complete orthonormal system of $L^{2}\left((-1,1),\left(1-x^{2}\right)^{\lambda-1 / 2} d x\right)$. It follows from (13) and (14) that for $\varphi \in L^{2}\left((-1,1),\left(1-x^{2}\right)^{\lambda-1 / 2} d x\right)$

$$
(\Phi \varphi)(z)=\sum_{m \in \mathbf{N}_{0}}\left(\varphi, \phi_{m}^{\lambda}\right) u_{m}^{\lambda}(z), \quad z \in B
$$

Hence, we have

$$
\|\Phi \varphi\|^{2}=\sum_{m \in \mathbf{N}_{0}}\left|\left(\varphi, \phi_{m}^{\lambda}\right)\right|^{2}=\|\varphi\|^{2}, \quad \Phi \phi_{m}^{\lambda}=u_{m}^{\lambda}
$$

which imply that $\Phi$ is unitary. 
Remark 2 Let $n$ be a positive integer that $n \geq 3$, and let $S^{n-1}$ be the $(n-1)$-dimensional unit sphere. Set $e_{1}={ }^{t}(1,0, \ldots, 0) \in S^{n-1}$. Then the identification $S O(n) / S O(n-1) \cong S^{n-1}$ is given by the mapping $g S O(n-1) \mapsto g e_{1}, g \in S O(n)$. On the other hand, in the case of $\lambda=(n-2) / 2$, the Gegenbauer polynomials $C_{m}^{\lambda}, m=0,1,2, \ldots$, give the zonal spherical functions on $(S O(n), S O(n-1))$. More precisely, for each $m \in \mathbf{N}_{0}$ define

$$
\varphi_{m}(g)=\frac{C_{m}^{\lambda}\left(\left(g e_{1}, e_{1}\right)\right)}{C_{m}^{\lambda}(1)}, \quad g \in S O(n)
$$

where (, ) denotes the canonical inner product on the real vector space $\mathbf{R}^{n}$. Then $\left\{\varphi_{m} \mid m \in \mathbf{N}_{0}\right\}$ is equal to the set of all the zonal spherical functions on $(S O(n), S O(n-1))$. Let $d_{m}$ denote the degree of the representation corresponding to $\varphi_{m}$. Then the following holds.

$$
\sum_{m \in \mathbf{N}_{0}} d_{m} \varphi_{m}(g) z^{m}=\frac{1-z^{2}}{\left(1-2 z\left(g e_{1}, e_{1}\right)+z^{2}\right)^{\frac{n}{2}}}, \quad g \in S O(n), z \in B
$$

which does not equal to $\Phi(z, x)$. But, since these functions differ only by a constant, the difference between them is not essential.

Remark 3 We can generalize Theorem 4 to the case of the Jacobi polynomials $P_{m}^{(\alpha, \beta)}(x), m=0,1,2, \ldots$, which have the following generating function $(c f .[8])$ :

$$
\begin{aligned}
& \sum_{m \in \mathbf{N}_{0}} \frac{(2 m+\alpha+\beta+1)(\alpha+\beta+1)_{m}}{(\alpha+1)_{m}} P_{m}^{(\alpha, \beta)}(x) z^{m} \\
& \quad=\frac{(\alpha+\beta+1)(z+1)}{(1-z)^{\alpha+\beta+2}} F\left(\frac{\alpha+\beta+2}{2}, \frac{\alpha+\beta+3}{2} ; \alpha+1 ; \frac{2 z(x-1)}{(1-z)^{2}}\right), z \in B,-1<x<1
\end{aligned}
$$

Let $\alpha, \beta>0$. If we replace $L^{2}\left((-1,1),\left(1-x^{2}\right)^{\lambda-1 / 2} d x\right)$ by $L^{2}\left((-1,1),(1-x)^{\alpha}(1+x)^{\beta} d x\right), \rho_{\lambda}(t)$ by

$$
\rho_{\alpha, \beta}(t)=t^{\frac{\alpha+\beta-1}{2}} \int_{t}^{1} u^{-\frac{\alpha+\beta+1}{2}}(1-u)^{\beta-1} d u \int_{\frac{t}{u}}^{1} v^{-\frac{\beta-\alpha+1}{2}}(1-v)^{\beta-1} d v
$$

and $\Phi(z, x)$ by

$$
\frac{(\alpha+\beta+1) \Gamma(\alpha+\beta+1)(z+1)}{\sqrt{2^{\alpha+\beta+2} \pi} \Gamma(\alpha+1) \Gamma(\beta)(1-z)^{\alpha+\beta+2}} F\left(\frac{\alpha+\beta+2}{2}, \frac{\alpha+\beta+3}{2} ; \alpha+1 ; \frac{2 z(x-1)}{(1-z)^{2}}\right)
$$

then we can obtain the desired result. For more details, refer to [9].

\section{Generating Function that Defines Unitary Operator - The Case of Zonal Spherical Functions on $(U(n), U(n-1))$}

Let $n$ be a positive integer that $n \geq 3$. Denote by $S\left(\mathbf{C}^{n}\right)$ the unit sphere in $\mathbf{C}^{n}$ and set $e_{1}=$ ${ }^{t}(1,0, \ldots, 0) \in S\left(\mathbf{C}^{n}\right)$. Then we have the identification $U(n) / U(n-1) \cong S\left(\mathbf{C}^{n}\right)$ by the mapping $g U(n-1) \mapsto g e_{1}, g \in U(n)$. The zonal spherical functions on $(U(n), U(n-1))$ are concerned with the functions $G_{p q}^{n-1}, p, q \in \mathbf{N}_{0}$ which have the following generating function (see Theorem 1.1. in [10]):

$$
\left(1-2 \operatorname{Re}(x z)+|z|^{2}\right)^{1-n}=\sum_{p, q \in \mathbf{N}_{0}} G_{p q}^{n-1}(x) z^{p} \bar{z}^{q}, \quad x, z \in B
$$


More precisely, for each $p, q \in \mathbf{N}_{0}$ define

$$
\varphi_{p q}(g)=\frac{G_{p q}^{n-1}\left(\left(g e_{1}, e_{1}\right)\right)}{G_{p q}^{n-1}(1)}, \quad g \in U(n)
$$

where (, ) denotes the canonical inner product on the complex vector space $\mathbf{C}^{n}$. Then $\left\{\varphi_{p q} \mid p, q \in \mathbf{N}_{0}\right\}$ is equal to the set of all the zonal spherical functions on $(U(n), U(n-1))$. For the functions $\varphi_{p q}, p, q \in \mathbf{N}_{0}$, refer to [11]. In this section, we deal with a generating function of $\left\{\varphi_{p q} \mid p, q \in \mathbf{N}_{0}\right\}$ that defines a unitary operator. For more details, refer to [3].

\section{1. $L^{2}$-Space Associated with the Zonal Spherical Functions on $(U(n), U(n-1))$}

In what follows, $x$ is an element of the unit open disk $B$ in $\mathbf{C}$, and $d x$ is the Lebesgue measure on $B$ induced from the identification $\mathbf{C} \cong \mathbf{R}^{2}$. Let $L^{2}\left(B,\left(1-|x|^{2}\right)^{n-2} d x\right)$ be the Hilbert space of Lebesgue measurable functions $\varphi$ on $B$ with

$$
\|\varphi\|=\sqrt{\int_{B}|\varphi(x)|^{2}\left(1-|x|^{2}\right)^{n-2} d x}<\infty
$$

The inner product is given by

$$
(\varphi, \psi)=\int_{B} \varphi(x) \overline{\psi(x)}\left(1-|x|^{2}\right)^{n-2} d x, \quad \varphi, \psi \in L^{2}\left(B,\left(1-|x|^{2}\right)^{n-2} d x\right)
$$

The functions $G_{p q}^{n-1}, p, q \in \mathbf{N}_{0}$ form a complete orthogonal system of $L^{2}\left(B,\left(1-|x|^{2}\right)^{n-2} d x\right)$. Denote by $\phi_{p q}^{n}$ the normalization of $G_{p q}^{n-1}$ with respect to the norm of this space. That is,

$$
\phi_{p q}^{n}(x)=\sqrt{\frac{(p+q+n-1) p ! q !(\Gamma(n-1))^{2}}{\pi \Gamma(p+n-1) \Gamma(q+n-1)}} G_{p q}^{n-1}(x)
$$

Then the system $\left\{\phi_{p q}^{n} \mid p, q \in \mathbf{N}_{0}\right\}$ is a complete orthonormal system of $L^{2}\left(B,\left(1-|x|^{2}\right)^{n-2} d x\right)$.

\subsection{Hilbert Space of Analytic Functions}

Let $\rho_{n}$ be the function on the direct product of the open interval $(0,1)$ with itself defined by

$$
\rho_{n}(u, v)=(u v)^{(n-3) / 2} \int_{1}^{\min (1 / u, 1 / v)} \frac{f_{n}(t u, t v)}{t} d t
$$

where

$$
f_{n}(u, v)=(u v)^{-(n-3) / 2}\{(1-u)(1-v)\}^{n-3}
$$

Let $\mathcal{F}_{n}$ be the space of analytic functions $f$ on $B^{2}$ in $\mathbf{C}^{2}$ with

$$
\|f\|=\sqrt{\int_{B^{2}}\left|f\left(z_{1}, z_{2}\right)\right|^{2} \rho_{n}\left(\left|z_{1}\right|^{2},\left|z_{2}\right|^{2}\right) d z_{1} d z_{2}}<\infty
$$

where $d z_{1}, d z_{2}$ are the Lebesgue measures on $B$ induced from the identification $\mathbf{C} \cong \mathbf{R}^{2}$. For $f \in \mathcal{F}_{n}$, we have the following inequality

$$
|f(z)| \leq\|f\| \sqrt{\sum_{p, q \in \mathbf{N}_{0}} \frac{(p+q+n-1) \Gamma(p+n-1) \Gamma(q+n-1)}{\pi^{2} p ! q !(\Gamma(n-2))^{2}}\left|z_{1}^{2 p} z_{2}^{2 q}\right|}, \quad z=\left(z_{1}, z_{2}\right) \in B^{2}
$$


which implies that $\mathcal{F}_{n}$ is a Hilbert space with inner product

$$
\langle f, g\rangle=\int_{B^{2}} f\left(z_{1}, z_{2}\right) \overline{g\left(z_{1}, z_{2}\right)} \rho_{n}\left(\left|z_{1}\right|^{2},\left|z_{2}\right|^{2}\right) d z_{1} d z_{2}, \quad f, g \in \mathcal{F}_{n}
$$

Set

$$
u_{p q}^{n}(z)=\sqrt{\frac{(p+q+n-1) \Gamma(p+n-1) \Gamma(q+n-1)}{\pi^{2} p ! q !(\Gamma(n-2))^{2}}} z_{1}^{p} z_{2}^{q}, \quad p, q \in \mathbf{N}_{0}, z=\left(z_{1}, z_{2}\right) \in B^{2}
$$

Then the system $\left\{u_{p q}^{n} \mid p, q \in \mathbf{N}_{0}\right\}$ is a complete orthonormal system of $\mathcal{F}_{n}$. It also follows from (15) that the space $\mathcal{F}_{n}$ has the reproducing kernel. The kernel is given by

$$
g_{w}^{n}(z)=\sum_{p, q \in \mathbf{N}_{0}} u_{p q}^{n}(\bar{w}) u_{p q}^{n}(z), \quad z, w \in B^{2}
$$

that is, $f(w)=\left\langle f, g_{w}^{n}\right\rangle$ for $f \in \mathcal{F}_{n}$ and $w \in B^{2}$.

\subsection{Unitary Operator Associated with the Zonal Spherical Functions on $(U(n), U(n-1))$}

A unitary operator on $L^{2}\left(B,\left(1-|x|^{2}\right)^{n-2} d x\right)$ to $\mathcal{F}_{n}$ is defined as follows. First, set

$$
\Phi(z, x)=\frac{(n-2)(n-1)}{\pi^{3 / 2}} \frac{1-z_{1} z_{2}}{\left(1-x z_{1}-\bar{x} z_{2}+z_{1} z_{2}\right)^{n}}, \quad z=\left(z_{1}, z_{2}\right) \in B^{2}, x \in B
$$

This has the following expansion:

$$
\Phi(z, x)=\sum_{p, q \in \mathbf{N}_{0}} u_{p q}^{n}(z) \phi_{p q}^{n}(x), \quad z \in B^{2}, x \in B
$$

which means that the function $\Phi(z, x)$ is a generating function of the zonal spherical functions on $(U(n), U(n-1))$.

For $\varphi \in L^{2}\left(B,\left(1-|x|^{2}\right)^{n-2} d x\right)$ we define

$$
(\Phi \varphi)(z)=\int_{B} \Phi(z, x) \varphi(x)\left(1-|x|^{2}\right)^{n-2} d x, \quad z \in B^{2}
$$

Then $\Phi \varphi \in \mathcal{F}_{n}$ and we have

Theorem 5 The operator $\Phi$ on $L^{2}\left(B,\left(1-|x|^{2}\right)^{n-2} d x\right)$ to $\mathcal{F}_{n}$ is unitary.

As in Gegenbauer's case, we positively use in our proof the fact that the system $\left\{\phi_{p q}^{n} \mid p, q \in \mathbf{N}_{0}\right\}$ is a complete orthonormal system of $L^{2}\left(B,\left(1-|x|^{2}\right)^{n-2} d x\right)$. It follows from (16), (17) and $\overline{\phi_{p q}^{n}(x)}=\phi_{q p}^{n}(x)$ that for $\varphi \in L^{2}\left(B,\left(1-|x|^{2}\right)^{n-2} d x\right)$

$$
(\Phi \varphi)(z)=\sum_{p, q \in \mathbf{N}_{0}}\left(\varphi, \phi_{q p}^{n}\right) u_{p q}^{n}(z), \quad z \in B^{2}
$$

Hence, we have

$$
\|\Phi \varphi\|^{2}=\sum_{p, q \in \mathbf{N}_{0}}\left|\left(\varphi, \phi_{p q}^{n}\right)\right|^{2}=\|\varphi\|^{2}, \quad \Phi \phi_{q p}^{n}=u_{p q}^{n}
$$

which imply that $\Phi$ is unitary. 
Remark 4 Let $d_{p q}$ denote the degree of the representation corresponding to $\varphi_{p q}$. Then the following holds.

$$
\sum_{p, q \in \mathbf{N}_{0}} d_{p q} \varphi_{p q}(g) z_{1}^{p} z_{2}^{q}=\frac{1-z_{1} z_{2}}{\left(1-z_{1}\left(g e_{1}, e_{1}\right)-z_{2}\left(e_{1}, g e_{1}\right)+z_{1} z_{2}\right)^{n}}, \quad g \in U(n), z_{1}, z_{2} \in B
$$

which does not equal to $\Phi(z, x)$. But, since these functions differ only by a constant, the difference between them is not essential.

\section{Group Theoretical Approach to Generating Functions that Define Unitary Operators}

Let $G$ be a compact connected Lie group, $K$ a closed subgroup of $G$, and $(G, K)$ a Riemannian symmetric pair of rank $\ell$ such that $G / K$ is simply connected. Then it is known (cf. [12]) that the set of all the zonal spherical functions on $(G, K)$ can be parametrized by $\mathbf{N}_{0}^{\ell}$. Denote the set by $\left\{\varphi_{m} \mid m \in \mathbf{N}_{0}^{\ell}\right\}$, and by $d_{m}$ the degree of the representation corresponding to $\varphi_{m}$. Further, we can assume that there exists a measure $\mu_{0}$ on $[0,1)^{\ell}$ such that

$$
d_{m}^{-1}=\int_{[0,1)^{\ell}} t^{m} d \mu_{0}(t), \quad m \in \mathbf{N}_{0}^{\ell} .
$$

See Lemma 2 in [5]. Weyl's dimension formula and Theorem 1 in [13] play important roles in the proof of (18). (Note that Theorem 1 in [13] gives a necessary and sufficient condition for a multisequence $\left\{\alpha_{m}\right\}_{m \in \mathbf{N}_{0}^{\ell}}$ to have a measure $\mu_{1}$ on $[0,1]^{\ell}$ such that $\alpha_{m}=\int_{[0,1]^{\ell}} t^{m} d \mu_{1}(t), m \in \mathbf{N}_{0}^{\ell}$.) Furthermore, it follows from (18) that we can construct a generating function of the form of (1) which defines a unitary operator.

\section{1. $L^{2}$-Space Associated with the Zonal Spherical Functions on $(G, K)$}

Let $d g$ be the normalized Haar measure on $G$, that is, it has the property that $\int_{G} d g=1$. Let $L^{2}(G)$ denote the Hilbert space of measurable functions $\varphi$ on $G$ with

$$
\|\varphi\|=\sqrt{\int_{G}|\varphi(g)|^{2} d g}<\infty
$$

The inner product is given by

$$
(\varphi, \psi)=\int_{G} \varphi(g) \overline{\psi(g)} d g
$$

Let $L^{2}(G, K)$ be the space of functions $\varphi \in L^{2}(G)$ which are bi-invariant under $K$. Then $L^{2}(G, K)$ is a closed subspace of $L^{2}(G)$, that is, $L^{2}(G, K)$ is also a Hilbert space with inner product (19). As is well known (cf. [12], [14]), the system $\left\{\varphi_{m} \mid m \in \mathbf{N}_{0}^{\ell}\right\}$ is a complete orthogonal system of $L^{2}(G, K)$, and has the following orthogonality relation:

$$
\int_{G} \varphi_{m}(g) \overline{\varphi_{m^{\prime}}(g)} d g=\left\{\begin{array}{cc}
d_{m}^{-1}, & m=m^{\prime} \\
0, & m \neq m^{\prime}
\end{array}\right.
$$

Set

$$
\phi_{m}=\sqrt{d_{m}} \varphi_{m}, \quad m \in \mathbf{N}_{0}^{\ell}
$$

Then the system $\left\{\phi_{m} \mid m \in \mathbf{N}_{0}^{\ell}\right\}$ is a complete orthonormal system of $L^{2}(G, K)$. 


\subsection{Hilbert Space of Analytic Functions}

It follows from (18) that we can construct a measure $\mu$ on $B^{\ell}$ such that

$$
\int_{B^{\ell}} z^{m} \overline{z^{m^{\prime}}} d \mu(z)=\left\{\begin{array}{cc}
d_{m}^{-1}, & m=m^{\prime} \\
0, & m \neq m^{\prime}
\end{array}\right.
$$

Let $\mathcal{F}$ be the space of analytic functions $f$ on $B^{\ell}$ with

$$
\|f\|=\sqrt{\int_{B^{\ell}}|f(z)|^{2} d \mu(z)}<\infty
$$

For $f \in \mathcal{F}$, we have the following inequality

$$
|f(z)| \leq\|f\| \sqrt{\sum_{m \in \mathbf{N}_{0}^{\ell}} d_{m}\left|z^{m}\right|^{2}}, \quad z \in B^{\ell}
$$

Note that $\sum_{m \in \mathbf{N}_{0}^{\ell}} d_{m}\left|z^{m}\right|^{2}<\infty$ for all $z \in B^{\ell}$, because $d_{m}$ is a polynomial of $m_{1}, \ldots, m_{\ell}$. The inequality (20) implies that $\mathcal{F}$ is a Hilbert space with inner product

$$
\langle f, g\rangle=\int_{B^{\ell}} f(z) \overline{g(z)} d \mu(z), \quad f, g \in \mathcal{F}
$$

It also follows from (20) that the space $\mathcal{F}$ has the reproducing kernel. That is, for each $w \in B^{\ell}$ there exists a unique $g_{w} \in \mathcal{F}$ such that $f(w)=\left\langle f, g_{w}\right\rangle$ for $f \in \mathcal{F}$. Set

$$
u_{m}(z)=\sqrt{d_{m}} z^{m}, \quad m \in \mathbf{N}_{0}^{\ell}, z \in B^{\ell}
$$

Then the system $\left\{u_{m} \mid m \in \mathbf{N}_{0}^{\ell}\right\}$ is a complete orthonormal system of $\mathcal{F}$. Note that the reproducing kernel of $\mathcal{F}$ has the following expansion:

$$
g_{w}(z)=\sum_{m \in \mathbf{N}_{0}^{\ell}} u_{m}(\bar{w}) u_{m}(z), \quad z, w \in B^{\ell}
$$

7.3. Unitary Operator Associated with the Zonal Spherical Functions on $(G, K)$

A unitary operator on $L^{2}(G, K)$ to $\mathcal{F}$ is defined as follows. First, set

$$
\Phi(z, g)=\sum_{m \in \mathbf{N}_{0}^{\ell}} d_{m} \varphi_{m}(g) z^{m}, \quad z \in B^{\ell}, g \in G
$$

This has the following expansion:

$$
\Phi(z, g)=\sum_{m \in \mathbf{N}_{0}^{\ell}} u_{m}(z) \phi_{m}(g), \quad z \in B^{\ell}, g \in G
$$

For $\varphi \in L^{2}(G, K)$ we define

$$
(\Phi \varphi)(z)=\int_{G} \Phi\left(z, g^{-1}\right) \varphi(g) d g, \quad z \in B^{\ell}
$$

Then $\Phi \varphi \in \mathcal{F}$ and we have 
Theorem 6 The operator $\Phi$ on $L^{2}(G, K)$ to $\mathcal{F}$ is unitary.

As stated above, the system $\left\{u_{m} \mid m \in \mathbf{N}_{0}^{\ell}\right\}$ is a complete orthonormal system of $\mathcal{F}$. Thus, it follows from (21), (22) and $\varphi_{m}\left(g^{-1}\right)=\overline{\varphi_{m}(g)}$ that for $\varphi \in L^{2}(G, K)$

$$
(\Phi \varphi)(z)=\sum_{m \in \mathbf{N}_{0}^{\ell}}\left(\varphi, \phi_{m}\right) u_{m}(z), \quad z \in B^{\ell}
$$

Hence, we have

$$
\|\Phi \varphi\|^{2}=\sum_{m \in \mathbf{N}_{0}^{\ell}}\left|\left(\varphi, \phi_{m}\right)\right|^{2}=\|\varphi\|^{2}, \quad \Phi \phi_{m}=u_{m}
$$

which imply that $\Phi$ is unitary.

Remark 5 The pair $(S O(n), S O(n-1))$ is a typical example of Theorem 6.

Remark 6 Suppose that a pair $(G, K)$ is a Gelfand pair of the compact type, and that the set of all the zonal spherical functions on $(G, K)$ is parametrized by $\mathbf{N}_{0}^{\ell}$, where $\ell$ is a positive integer. And as in the first part of this section, define $\left\{\varphi_{m} \mid m \in \mathbf{N}_{0}^{\ell}\right\}$ and $d_{m}\left(m \in \mathbf{N}_{0}^{\ell}\right)$. If the pair $(G, K)$ has a measure $\mu_{0}$ on $[0,1)^{\ell}$ which satisfies (18), and the series $\sum_{m \in \mathbf{N}_{0}^{\ell}} d_{m}\left|z^{m}\right|^{2}$ converges for each $z \in B^{\ell}$, then Theorem 6 also holds. The pair $(U(n), U(n-1))$ is such an example.

\section{References}

1. Bargmann, V. On a Hilbert space of analytic functions and an associated integral transform Part I. Comm. Pure Appl. Math. 1961, 14, 187-214.

2. Watanabe, S. Hilbert spaces of analytic functions and the Gegenbauer polynomials. Tokyo J. Math. 1990, 13, 421-427.

3. Watanabe, S. Spherical harmonics on $U(n) / U(n-1)$ and associated Hilbert spaces of analytic functions. Tokyo J. Math. 2000, 23, 351-360.

4. Essadiq, A. $q$-analogue of the Watanabe unitary transform associated to the $q$-continuous Gegenbauer polynomials. Lett. Math. Phys. 2000, 53, 233-242.

5. Watanabe, S. Symmetric pairs, Gelfand pairs and pairs such that generating functions of zonal spherical functions define unitary operators-the case of the compact type. J. Funct. Anal. 2006, 238, 427-446.

6. Fock, V. Verallgemeinerung und Lösung der Diracschen statistischen Gleichung. Z. Phys. 1928, 49, 339-357.

7. Temme, N.M. Special Functions, An Introduction to the Classical Functions of Mathematical physics; John Wiley \& Sons, Inc.: New York, NY, USA, 1996.

8. Prudnikov, A.P.; Brychkov, Y.A.; Marichev, O.I. Integrals and Series; Gordon and Breach: New York, NY, USA, 1986.

9. Watanabe, S. Generating functions of the Jacobi polynomials and related Hilbert spaces of analytic functions. Proc. Japan Acad. 1998, 74A, 46-48. 
10. Watanabe, S. Generating functions and integral representations for the spherical functions on some classical Gelfand pairs. J. Math. Kyoto Univ. 1993, 33, 1125-1142.

11. Johnson, K.D.; Wallach, N.R. Composition series and intertwining operators for the spherical principal series I. Trans. Amer. Math. Soc. 1977, 229, 137-173.

12. Takeuchi, M. Modern Spherical Functions; Amer. Math. Soc.: Providence, RI, USA, 1994.

13. Hildebrandt, T.H.; Schoenberg, I.J. On linear functional operations and the moment problem for a finite interval in one or several dimensions. Ann. of Math. 1933, 34, 317-328.

14. Helgason, S. Groups and Geometric Analysis; Academic Press: New York, NY, USA, 1984.

(c) 2010 by the author; licensee Molecular Diversity Preservation International, Basel, Switzerland. This article is an open-access article distributed under the terms and conditions of the Creative Commons Attribution license http://creativecommons.org/licenses/by/3.0/. 